Rakenteiden Mekaniikka (Journal of Structural Mechanics)

Vol. 54, No. 2, 2021, pp. 95-116

http://rakenteidenmekaniikka.journal.fi/index

https://doi.org/10.23998/rm.85711

(C) 2021 The Authors

Open access under license CC BY 4.0

\title{
Buckling analysis of members restrained by sandwich panels
}

\author{
Markku Heinisuo
}

Summary An analytic method is presented for the analysis of flexural restraint of members by sandwich panels. Using the method, which is based on the solutions of the fourth order differential equations, the restraint effect of sandwich panels can be approximated in practical cases. The reliability of the method is shown based on tests and finite element analyses. New results are shown using the analytic method for buckling cases and for $P-\delta$ analysis in the elastic range. The exact finite element method (FEM) formulation is given for more complicated cases.

Key words: sandwich panels, flexural restraint, flexural buckling of restrained members, $P-\delta$ effect of restrained members, exact FEM

Received: 25 March 2020. Accepted: 14 June 2021. Published online: 14 June 2021.

\section{Introduction}

Sandwich panels which are composed of thin metal faces with mineral wool, rock wool, PUR, PIR, etc. cores, see Figure 1, are widely used in buildings due to their good thermal insulation and air tightness providing energy efficient solutions for walls and roofs and finally for entire buildings.

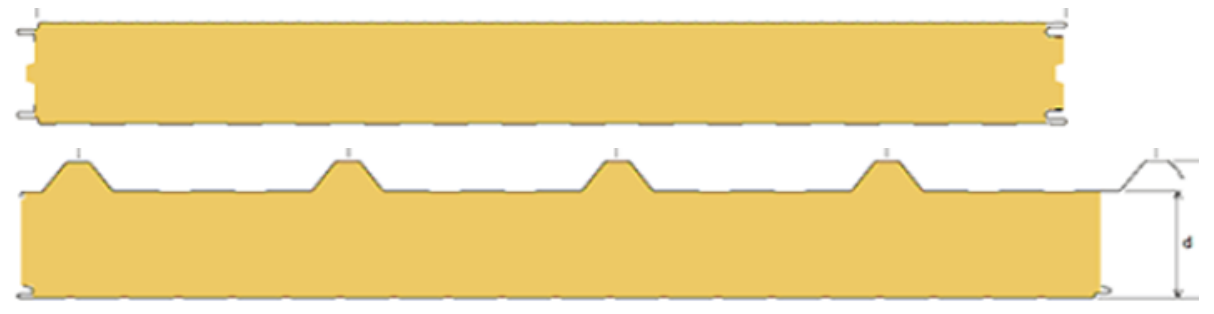

Figure 1. Typical sandwich panels.

In the walls the panels may be vertical or horizontal. Typical layout of the wall is such that one span horizontal panels are from column to column and connected to the 
columns with long screws. In Figure 2 is shown a large boiler building where this technique has been used.

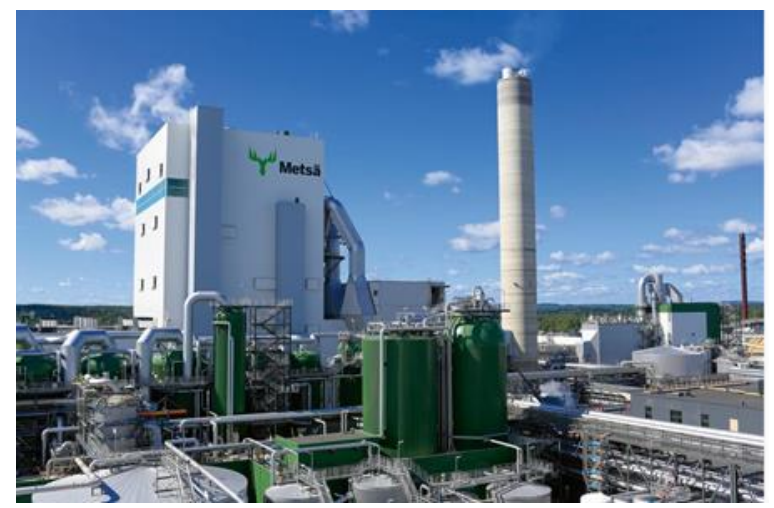

Figure 2. Boiler building, Metsä Group, Äänekoski, Finland. White and light blue walls of the large building are made using horizontal sandwich panels from column to column (photo: https://www.ruukki.com/fin/b2b/referenssit/reference-details/mets\%C3\%A4-groupbiotuotetehdas-\%C3\%A4\%C3\%A4nekoski)

In Figure 3 is shown a typical detail of the horizontal wall panels which are connected to the columns.

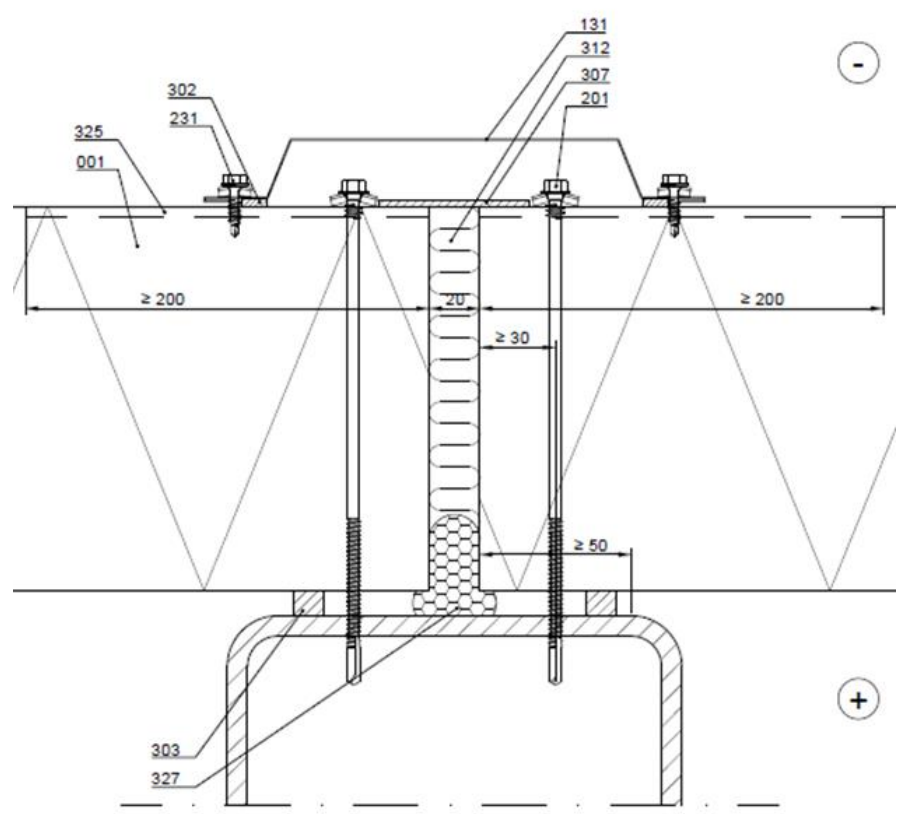

Figure 3. Detail of horizontal wall panel. Gap and insulation between panels number 312. Long screws which connect panels to member number 201. Not structural parts, numbers 131, 312, 307, 320, 231, 303, 327. (more details: https://cdn.ruukki.com/docs/default-source/b2bdocuments/sandwich-panels/spa-energyulkosein\%c3\%a4detaljit_20170504.pdf?sfvrsn=10eb8384_15) 
The long screws (number 201 in Figure 3) connect the panels to the tubular member. Traditionally the tubular member is considered as a supporting structure for the panels. However, in the present study the panels support the tubular member. If the steel member is not susceptible to torsional deformations, such as rectangular hollow sections (RHSs) and some I-sections, then the sandwich panels restraint the member for flexural deformations in the plane of the wall. This restraint effect may just reduce the displacements and stresses of the member when the member is loaded in the plane of the wall, e.g. wind load of a corner column of the building. One important case is the buckling of the member in the plane of the wall, normally weak axis buckling. It is believed by the author that this stabilizing effect of sandwich panels include widely unused potential to cost and $\mathrm{CO}_{2}$-emission efficient buildings.

The scope of this paper falls into the stressed skin design. Interest in the stressed skin design dates to the early 1950s when it was found that stresses and deflections of steel frames were smaller using the stressed skin design than predicted by the usual design calculations. The stressed skin design has gained attention especially in the design of single-storey industrial buildings due to possible cost savings when stabilizing distinct members and even the whole building. The first book on the subject was published in [2] and European recommendations for the stressed skin design were published in [6]. Design manuals are now available in [4] and [16]. The stressed skin design is briefly referred in standards such as [9] and it can be considered as a wellknown technique in the structural design. In these references the stressed skin is mainly considered as a trapezoidal sheeting which restraints the member in the plane of the sheeting. The theory of Winkler foundation has been used in Ref. [16] to describe the local response of the trapezoidal sheeting for the loaded member. In this theory it is supposed that the sheeting itself is rigid and all deformations take place at the connections between the sheeting and the member.

The early researches were dealt with the stressed skin design where the trapezoidal sheeting is used for stabilizing. Similar savings, as with the trapezoidal sheeting, may be achieved with sandwich panels. The shear stiffness of sandwich panels was derived and validated by tests in Germany in 1990's [1] for the panels and connectors which are frequently used in practice, see the validity range of the theory, which is given later. The theory and corresponding differential equation were developed in [12] and validated by tests. In this theory the restraint effect of sandwich panels can be considered for the flexural buckling and for the lateral torsional buckling of the supported members. The restraint for flexural deformations of the member is in the scope of this paper. The torsional restraint is not considered here.

The theory of [12] was studied extensively in a European project EASIE [5] leading to the design recommendations [7]. In [17] are presented the FEM simulations and the results were in the good agreement with the tests of [12]. In [11] are presented analytical solutions of flexural buckling cases and parametric studies with different properties of panels and members. The theory leads to the fourth order differential equations with or without axial force. The solutions of the differential equations can be derived based on classical theory of differential equations.

The theory of [12] is based on following assumptions (the supported member is vertical): 
1. The connected panel is staying in the horizontal position.

2. Longitudinal panel joints do not restraint the longitudinal forces between panels.

3. All deformations take place at the connections and the panel itself is supposed to behave as a rigid body. The shear deformations and resistances of the connectors are defined based on the design manual [7].

4. Connectors at the ends of the panels form the moments, which resist the rotation of the member between connectors.

5. The curvature of the member is supposed to be small between connectors.

6. The elastic theory is supposed to be valid for panels, connectors and members.

7. The axial force of the member is considered in the equilibrium of the member meaning exact $P-\delta$ analysis with small rotations.

8. The panel and the connector are supposed to act at the axis of the member neglecting the eccentricity of the joint. The motivation to this assumption is based on that the member is not susceptible to torsional deformations.

The assumption number 4 introduces the effects of panels to the equilibrium equation of the member.

The validity range of the theory is the same as is given in [7] and [12]. The theory covers sandwich panels with steel or aluminium faces and a core made of polyurethane (PUR)-, polyisocyanurate (PIR)- and EPS-foam or made of mineral wool. The face can be corrugated or flat, see Figure 1. The core thicknesses of the faces should be $0.40-$ $1.00 \mathrm{~mm}$. The minimum thickness of the panel is $40 \mathrm{~mm}$. The supported steel member should not be susceptible to torsional deformations, such as rectangular hollow sections and some I-sections. Maximum size of the I-section given in [12] is HEB300. The core thickness of the supported steel member should be larger than $1.5 \mathrm{~mm}$. The nominal diameters of the self-drilling or self-tapping screws should be $5.5-8.0 \mathrm{~mm}$.

When we use the exact solutions of the differential equations with the axial force of the member then we can follow so called $P-\delta$ analysis exactly in the elastic range. This kind of analysis is well established for the homogeneous elastic members without the stressed skin action since [19] and by many others. The exact finite element formulations (Note. In [3] the term "direct-stiffness method" is used instead of "exact FEM formulation”) enable the use of only one ,natural“ finite element per member if the properties of the member or the supporting structure do not change within the element, often within spans. This means that in the global analysis minimum amounts of finite elements are needed when using the natural elements and this feature is especially important in the optimization of structures. For the members without stressed skin design this kind of exact finite element formulation is presented in [18] and with shear deformations in [3]. Exact FEM formulations have been presented for members on elastic Winkler foundation in [13] and with the axial load in [8]. The exact FEM formulations using the solutions of the differential equations of [12] have not been presented in the literature before this study.

When using the exact FEM formulation with the axial force then the corresponding eigenvalues can be derived using the algorithm of [20] as is done e.g. in [18]. It was stated in [10] that the algorithm of [20] made it possible to use the exact FEM formulation generally in the eigenvalue problems. 
The scope of this paper is an analytic method for flexural stabilization of members by sandwich panels. The analytic method enables simple calculations in the design of restrained members, instead of heavier finite element calculations. The analytical exact results are valuable in practical engineering whenever they are available.

The theory, which is based on these assumptions and solutions of the governing differential equations [12], are the basis of the analytic method, which is considered in this paper. Similarities and differences compared to the theory of members without restraints by the panels are outlined.

The structure of this paper is as follows. In the chapter 1 are given comparisons between results of tests [12], FEM [17] and the analytic method aiming to demonstrate the usability of the analytic method in real structures. In the chapter 2 buckling of four Euler cases are presented and the comparisons are given with FEM. Formerly, only the simply supported member [12] and the cantilever [11] have been presented in the literature. New solutions of the corresponding differential equation enable to perform the exact $P-\delta$ analysis in the elastic range. In the chapter 3 the exact FEM formulation is given.

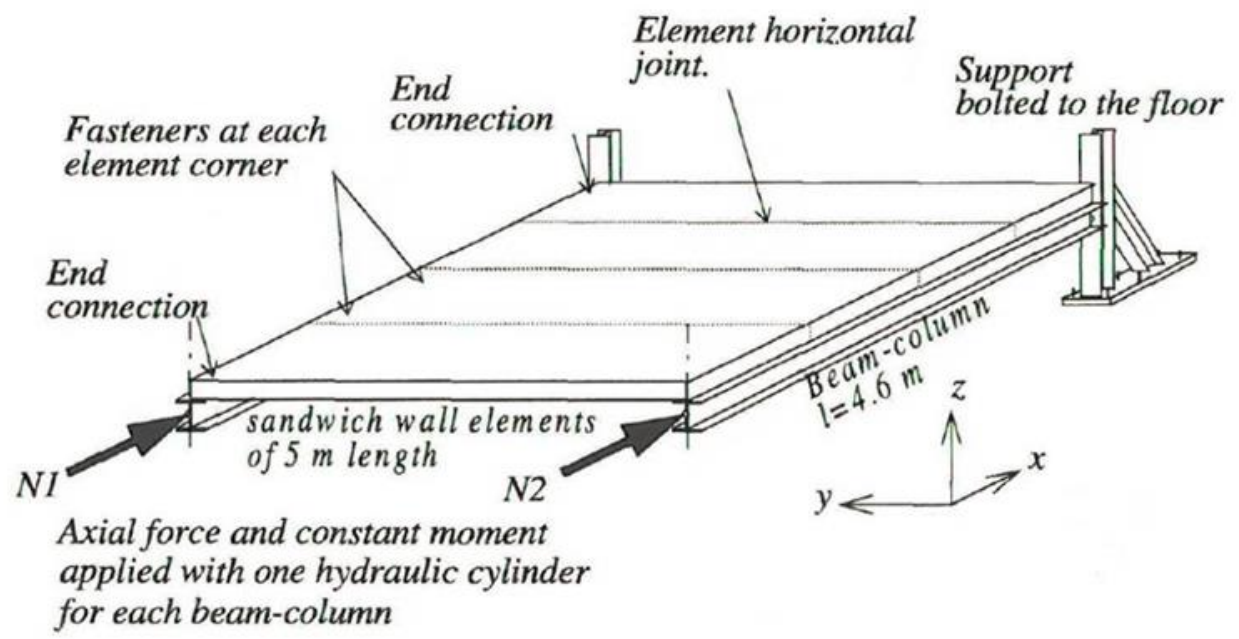

Figure 4. Experimental set up [12].

\section{Simply supported member}

Consider two simply supported (simply supported both against strong and weak axis bending) HEA120 (steel grade S275) members with spans $4.62 \mathrm{~m}$. The members are supported with four $150 \mathrm{~mm}$ thick and $5 \mathrm{~m}$ long sandwich panels, as is shown in Figure 4. The nominal thicknesses of faces are $0.6 \mathrm{~mm}$, the width of one panel is $B=1.1 \mathrm{~m}$. The ultimate strength of the faces is estimated as $420 \mathrm{MPa}$ (measured yield strength 390 $\mathrm{MPa}$ ). The panels are connected to the flanges of the members by screws of $5.5 \mathrm{~mm}$ diameter at the corners of the panels with edge distance $30 \mathrm{~mm}$ in both directions. This case has been studied experimentally in [12] and by FEM in [17]. 
The differential equation of the flexural member which is restrained by the sandwich panels is for a "large" axial compressive force $N$ of the member [12]

$$
\frac{d^{4} v}{d x^{4}}+\lambda^{2} \frac{d^{2} v}{d x^{2}}=\frac{q}{E I}
$$

where $v$ is the deflection of the member in $y$-direction in Figure 4, $x$ is the longitudinal axis of the member, $q$ is the distributed force along the member in y-direction, $E I$ is the bending stiffness of the member in weak axis of the member and $k_{v}$ is the shear stiffness of one connector, generally see [7], $c_{k}$ is the distance of symmetric connectors at the ends of panels and there exist $n$ pairs of connectors at the ends of panels. In the case of Figure 4 the number $n=1, B=1100 \mathrm{~mm}$ and $c_{1}=1100-30-30=1040 \mathrm{~mm}$. The slenderness $\lambda$ and the "large" axial force $N$ are defined in Eq. (2).

$$
\lambda=\sqrt{\frac{N}{E I}-\frac{k_{v}}{2 B E I} \sum_{k=1}^{n} c_{k}^{2}} \quad ; \quad N \geq \frac{k_{v}}{2 B} \sum_{k=1}^{n} c_{k}^{2}
$$

If the axial force is "small" then

$$
\frac{d^{4} v}{d x^{4}}-\bar{\lambda}^{2} \frac{d^{2} v}{d x^{2}}=\frac{q}{E I}
$$

where

$$
\bar{\lambda}=\sqrt{\frac{k_{v}}{2 B E I} \sum_{k=1}^{n} c_{k}-\frac{N}{E I}} \quad ; \quad N<\frac{k_{v}}{2 B} \sum_{k=1}^{n} c_{k}^{2}
$$

The differential equations (1) and (3) are formally same as the equations for the members without restraints. The transition point where the governing equation changes is different, and it is dependent on the axial force $N$ as is shown in Eqs. (2) and (4). The difference is present, also, in the boundary conditions, and these are shown in detail in [11].

The sine function fulfills the boundary conditions of the deflections $v(x)$ in $y$ direction (weak axis bending), see Figure 4, $v(0)=v(L)=0$ ( $L$ is the span of the member) and for the bending moments $M(0)=M(L)=0$ at the supports for the simply supported case. Without the distributed load $q$ and with the "large" axial load $N$ the deflection is 


$$
v(x)=A_{r} \cdot \sin \left(\frac{r \pi x}{L}\right)
$$

where $r$ is an integer. Substituting Eq. (5) into the differential equation (1) gives

$$
\begin{aligned}
& E I \frac{r^{4} \pi^{4}}{L^{4}}-N \frac{r^{2} \pi^{2}}{L^{2}}+\frac{k_{v}}{2 \cdot B} \sum_{k=1}^{n} c_{k}^{2} \frac{r^{2} \pi^{2}}{L^{2}}=0 \Rightarrow \\
& N_{c r}=E I \frac{r^{2} \pi^{2}}{L^{2}}+\frac{k_{v}}{2 B} \sum_{k=1}^{n} c_{k}^{2}
\end{aligned}
$$

It can be seen in Eq. (7), that the first eigenmode $r=1$ gives the smallest $N_{c r}$ in this case. It can be seen, also, that the first part of $N_{c r}$ is the same as for the member without sandwich panels. The buckling length $L_{c r}$ in the plane of the panel can be solved from the Euler equation

$$
\frac{\pi^{2} E I}{L_{c r}^{2}}=N_{c r}=\frac{\pi^{2} \cdot E I}{L^{2}}+\frac{k_{v}}{2 B} \sum_{k=1}^{n} c_{k}^{2} \Rightarrow
$$

and

$$
L_{c r}=\frac{L}{\sqrt{1+\frac{k_{v}}{2 B} \cdot \sum_{k=1}^{n} c_{k}^{2} \cdot \frac{L^{2}}{\pi^{2} \cdot E \cdot I}}}
$$

Use the following data for the case of Figure 4:

- Young's modulus of the member: $E=210000 \mathrm{MPa}$;

- Moment of inertia HEA120, weak direction: $I=I_{z}=230.9 \mathrm{~cm}^{4}$;

- Moment of inertia, strong direction: $I_{y}=606.2 \mathrm{~cm}^{4}$;

- Area HEA120: $A=25.2 \mathrm{~cm}^{2}$;

- Measured yield strength of HEA120: $f_{y}=300 \mathrm{MPa}$;

- Span of the member: $L=4.62 \mathrm{~m}$;

- Shear stiffness of screw: $k_{v}=1.0 \mathrm{kN} / \mathrm{mm}$, given in [12];

- Width of one panel: $B=1.1 \mathrm{~m}$;

- Distance of screws: $c_{1}=1040 \mathrm{~mm}$.

The plastic resistance of the member is $N_{p l, R d}=756 \mathrm{kN}$. The buckling resistances for weak and strong axis buckling are without sandwich panels following [9] $171 \mathrm{kN}$ and $390 \mathrm{kN}$, respectively. Eqs. (8) and (9) give the results $N_{c r}=716 \mathrm{kN}$ and $L_{c r}=2586 \mathrm{~mm}$ 
implying $L_{c r} / L=0.56$ for the restrained member. The buckling load with the sandwich panels is close to the plastic resistance of the cross-section $\left(N_{c r} / N_{p l, R d}=0.95\right)$, indicating that the sandwich panels restraint effectively the buckling in the plane of the panels, and the buckling with respect to the major axis of the member (out of the plane of the panels) is critical.

The tests of [12] gave the ultimate axial load $412 \mathrm{kN}$ and the buckling occurred with respect to the major axis of the member (out of the plane of the panels). This result is very near by the buckling resistance of [9] $390 \mathrm{kN}$. In the tests small eccentricities of $L / 1000$ were present in both directions. The FEM calculations of [17] gave the result $419 \mathrm{kN}$. The FEM model included the same geometrical imperfections as the tests and measured [12] residual stresses of HEA120. The results are collected to Table 1.

Table 1. Results of simply supported beam.

\begin{tabular}{llc}
\hline Without & Plastic resistance $(\mathrm{kN})$ & 756 \\
panels & Buckling resistance, weak axis $(\mathrm{kN})$ & 171 \\
& Buckling resistance, strong axis $(\mathrm{kN})$ & 390 \\
& Weak axis buckling length $(\mathrm{m})$ & 4.620 \\
\hline \multirow{2}{*}{ With panels } & Buckling load, weak axis $(\mathrm{kN})$ & 716 \\
& Ultimate load, test $(\mathrm{kN})$ & 412 \\
& Ultimate load, FEM $(\mathrm{GMNIA})(\mathrm{kN})$ & 419 \\
& Weak axis buckling length $(\mathrm{m})$ & 2.586 \\
\hline
\end{tabular}

The shear stiffness of the screw was given in [12] and it was smaller $(1 \mathrm{kN} / \mathrm{mm})$ than given in the ECCS recommendations $(2.8 \mathrm{kN} / \mathrm{mm})$. The small value may be based on the smaller edge distance than is recommended to be used in ECCS. In the tests edge distances of screws with the diameter $5.5 \mathrm{~mm}$ was $30 \mathrm{~mm}$ in both directions. In the FEM results were detected some lateral-torsional displacements at the ultimate limit state [17], which were not reported in the tests [12]. Torsional restraints were not used in the FEM model, only the lateral restraints.

The tests and FEM analyses indicate the same result as the analytical solution: sandwich panels can provide full lateral support for the member against the weak axis buckling in this case. Without sandwich panels the axial resistance of the member is 171 $\mathrm{kN}$ (weak axis buckling). With sandwich panels, the axial resistance is $390 \mathrm{kN}$ (strong axis buckling). It is seen that the axial resistance of the member increases significantly, when the sandwich panels are used for stabilizing the member in this case.

The maximum shear force of the connector can be calculated supposing e.g. sine function for the deflection of the member with some maximum amplitude $e_{0}$ :

$$
v(x)=e_{0} \cdot \sin \left(\frac{\pi x}{L}\right)
$$

The shear force $F$ of the connector is related to the rotation of the member [11] 


$$
F=k_{v} \cdot \frac{c}{2} \cdot \frac{d v}{d x}
$$

The maximum values are at the end of the member

$$
F_{\max }=k_{v} \cdot \frac{c}{2} \cdot \frac{d v(0)}{d x}=k_{v} \cdot \frac{c}{2} \cdot e_{0} \cdot \frac{\pi}{L}
$$

Using the values for $k_{v}, c$, and $L$ from the above example, and setting $e_{0}=15 \mathrm{~mm}=$ $L / 300$, the maximum shear load of the connector according to Eq. (12) is $F_{\max }=5.3 \mathrm{kN}$. In Figure 5 are the FEM results of [17].

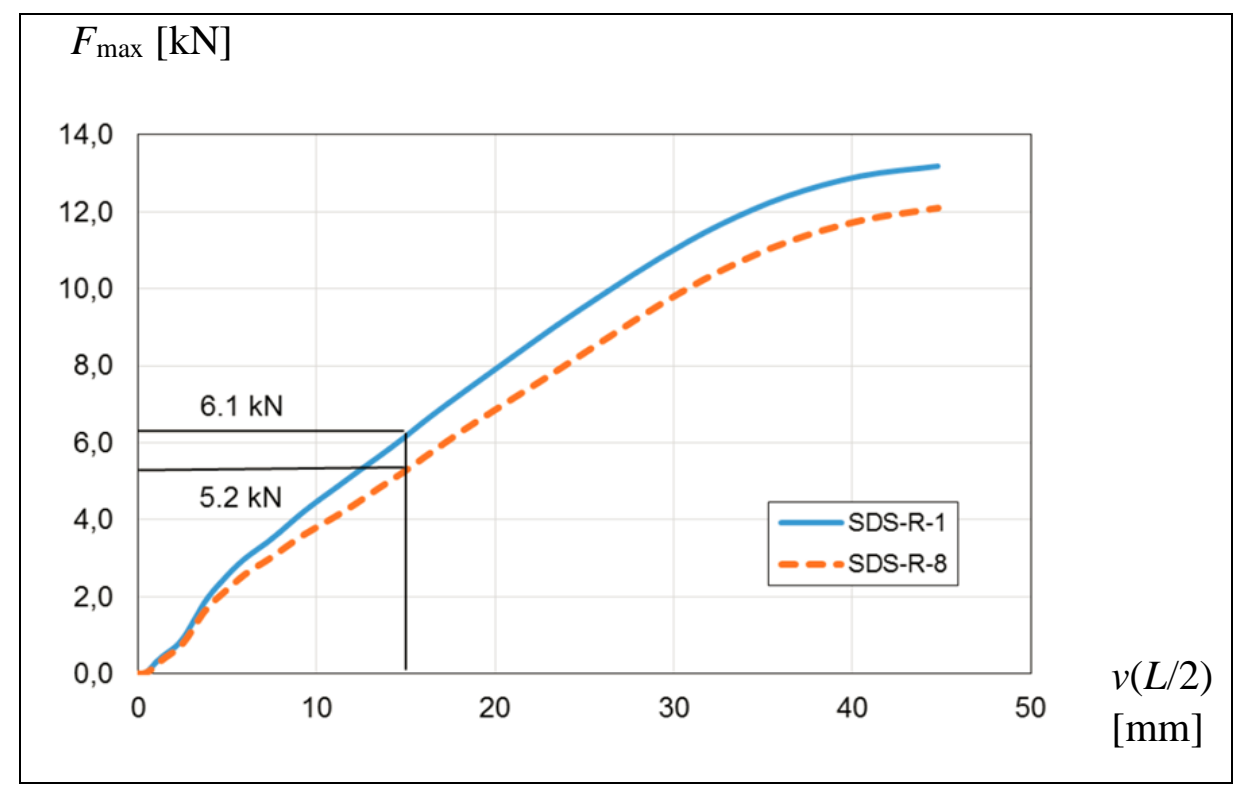

Figure 5. Maximum shear force of the connector versus the out-of-plane deflection at the midspan of the member, FEM results [17]. SDS-R-1 and SDS-R-8 denote two end screws connecting sandwich panels along the right column in the analyzed structure.

It can be seen in Figure 5, that the analytical solution (12) $5.3 \mathrm{kN}$ is close to the results obtained by FEM.

The axial forces of the member are plotted in Figure 6 as a function of the mid-span deflection in the direction of the $z$-axis (see Figure 4) based on FEM and tests. 


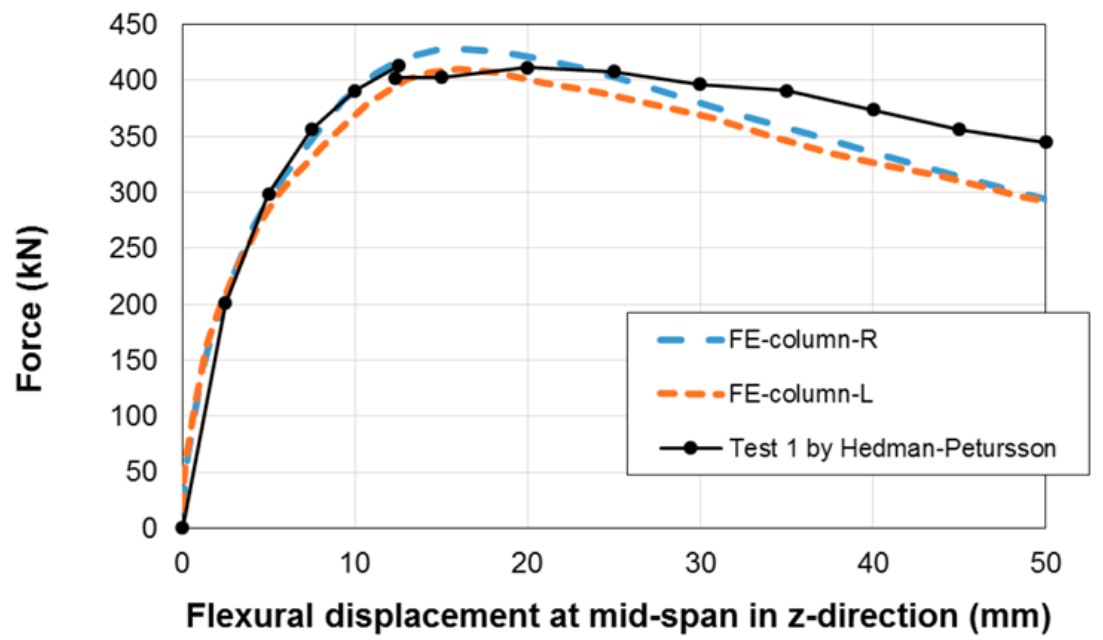

Figure 6. Axial force versus displacement, [17].

It can be seen in Figure 6 that when the deflection reaches about $15 \mathrm{~mm}$, the member fails. For this deflection, the maximum connector forces along the member according to Figure 5 are about $5-6 \mathrm{kN}$, which are larger than the shear resistance values given in [7]. In the tests of [12] no failures were reported at the connectors. Based on the FEM results, the connections seem to be very ductile.

It can be concluded that the analytic method works well with respect to the buckling load and the connector forces, which are the most important design properties in this case.

\section{Examples of exact solutions}

Consider four Euler flexural buckling cases. The buckling forces are solved from the eigenvalue problems of the differential equation (1). The solutions are coming from the boundary conditions. When the member length is $L$ then it is seen [11] that the boundary conditions for the restrained member lead to the same characteristic equations and corresponding lowest positive eigenvalues with the "large" compressive axial load. With the "small" axial load no eigenvalues are found. The characteristic equations and the lowest eigenvalues are:

- Fixed/Free: $\cos (\lambda L)=0 \Rightarrow(\lambda L)_{c r}=\pi / 2$,

- Hinged/Hinged: $\sin (\lambda L)=0 \Rightarrow(\lambda L)_{c r}=\pi$,

- Fixed/Hinged: $\tan (\lambda L)=\lambda L \Rightarrow(\lambda L)_{\mathrm{cr}}=\pi / 0.699$,

- Fixed/Fixed: $\cos (\lambda L)=1 \Rightarrow(\lambda L)_{c r}=2 \pi$.

From these we get the buckling loads $N_{c r}$ and the buckling lengths $L_{c r}$ for the restrained members 


$$
N_{c r}=\frac{\pi^{2} E I}{L_{c r, 0}^{2}}+\frac{k_{v}}{2 B} \sum_{k=1}^{n} c_{k}^{2}=\frac{\pi^{2} E I}{L_{c r}^{2}}
$$

and

$$
L_{c r}=\frac{L_{c r, 0}}{\sqrt{1+\frac{k_{v}}{2 B} \sum_{k=1}^{n} c_{k}^{2} \frac{L_{c r, 0}^{2}}{\pi^{2} E I}}}
$$

where $L_{c r, 0}$ is the buckling length without restraints.

Consider numerical examples of Euler cases. The member is a cold-formed rectangular hollow section CFRHS300x200x8 (weak axis buckling, $I=5042 \mathrm{~cm}^{4}, E=$ $210000 \mathrm{MPa}$ ), length $L=9.3 \mathrm{~m}$. The stiffness of one screw is $k_{v}=2.8 \mathrm{kN} / \mathrm{mm}$ based on [7] and one pair of screws at the end of panel is employed with $c_{1}=1 \mathrm{~m}(n=1)$ and the width of the panel is $B=1.2 \mathrm{~m}$. The results are shown in Table 2 . The results of discretized FEM are also shown. In the FEM analysis, see [11], the member is divided into 20 beam elements and the connections are modeled as horizontal springs. The lowest panel in FEM model is narrower $(B=0.9 \mathrm{~m})$ than 7 others.

Table 2. Flexural buckling loads and buckling lengths for weak axis buckling of the coldformed steel column CFRHS300*200*8, $L=9.3 \mathrm{~m}$, panels $B=1.2 \mathrm{~m}$, one pair of screws $c_{1}=1$ $\mathrm{m}, k_{v}=2.8 \mathrm{kN} / \mathrm{mm}$.

\begin{tabular}{lcccc}
\hline & Fixed/Free & Hinged/Hinged & Fixed/Hinged & Fixed/Fixed \\
\hline Analytic $N_{c r, 0}[\mathrm{kN}]$ & 302 & 1208 & 2466 & 4833 \\
FEM $N_{c r, 0}[\mathrm{kN}]$ & 302 & 1226 & 2517 & 4711 \\
Analytic $L_{c r, 0}[\mathrm{~m}]$ & 18.60 & 9.30 & 6.51 & 4.65 \\
Analytic $N_{c r}[\mathrm{kN}]$ & 1469 & 2375 & 3632 & 6000 \\
FEM $N_{c r}[\mathrm{kN}]$ & 1500 & 2334 & 3715 & 5862 \\
Analytic $L_{c r}[\mathrm{~m}]$ & 8.44 & 6.63 & 5.36 & 4.17 \\
$L_{c r} / L_{c r, 0}$ & 0.45 & 0.71 & 0.82 & 0.90 \\
$L_{c r} / L$ & 0.90 & 0.71 & 0.58 & 0.45 \\
$L_{c r, 0} / L$ & 2.00 & 1.00 & 0.70 & 0.50 \\
\hline
\end{tabular}

It is seen that all analytic and FEM results are near each other. The buckling load ratio of the cantilever (Fixed/Free in Table 2) with restraints to the buckling load without restraints is $1469 / 302=4.86$. The corresponding ratio for the case Fixed/Fixed is $6000 / 4833=1.24$. The ratios for other cases are between these extremes. It is seen that this ratio is increasing when the member is coming slenderer. In all cases the effects of restraints to the buckling loads and to the buckling lengths can be considered large. In Figure 7 are shown the buckling modes of four Euler cases using FEM. 


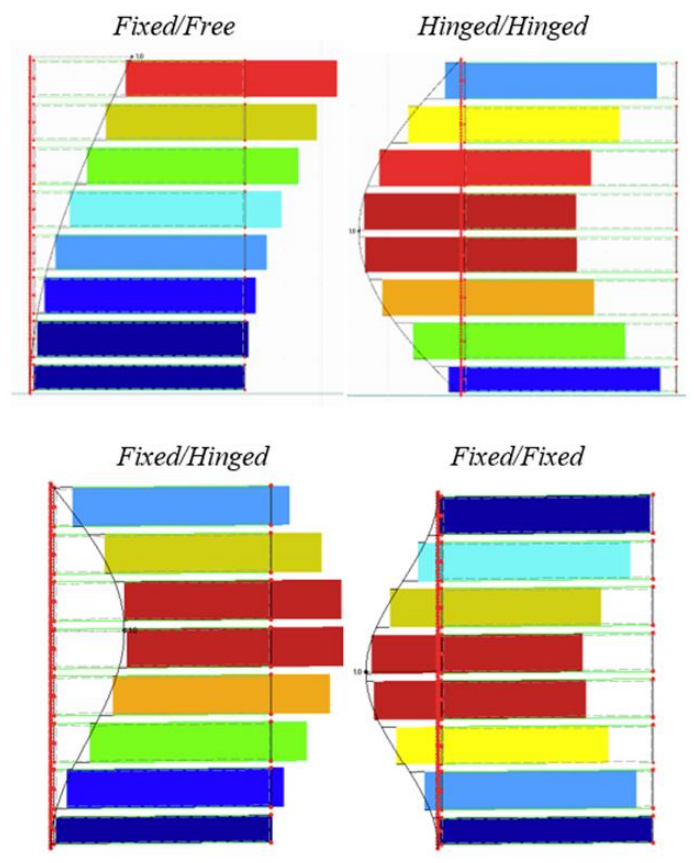

Figure 7. Buckling modes of Euler cases. Color bars illustrate separate panels.

Consider next an example of the exact $P-\delta$ analysis. The vertical cantilever of length $L$ is restrained with the sandwich panels and loaded by the uniform axial load $N$ and by the lateral load $F$ at the free end. The origin of longitudinal coordinate $x$ is at the base joint. The boundary conditions are:

$$
\begin{gathered}
v(0)=0 \\
\frac{d v(0)}{d x}=0 \\
M(L)=-E I \frac{d^{2} v(L)}{d x^{2}}=0 \\
Q(L)=-E I \frac{d^{3} v(L)}{d x^{3}}-\left(N-\frac{k_{v}}{2 B} \sum_{k=1}^{n} c_{k}^{2}\right) \frac{d v(L)}{d x}=F
\end{gathered}
$$

where $M$ and $Q$ are the moment and the shear force of the member. If the axial force is "large" then applying the boundary conditions (15) - (18) the deflection of the member is 


$$
v(x)=\frac{F}{\lambda^{2} E I}\left(-\frac{\sin \lambda x}{\lambda}+\frac{\tan \lambda L}{\lambda} \cos \lambda x+x-\frac{\tan \lambda L}{\lambda}\right)
$$

If the axial force is "small" then

$$
v(x)=\frac{F}{\bar{\lambda}^{2} E I}\left(\frac{\sinh \bar{\lambda} x}{\bar{\lambda}}-\frac{\tanh \bar{\lambda} L}{\bar{\lambda}} \cosh \bar{\lambda} x-x+\frac{\tanh \bar{\lambda} L}{\bar{\lambda}}\right)
$$

The maximum displacement is at the top of the member $x=L$. Use the same data as above, i.e. CFRHS300x200x8 (weak axis bending), $L=9.3 \mathrm{~m}, B=1.2 \mathrm{~m}, c_{1}=1 \mathrm{~m}, n=$ 1 . By varying the stiffness $k_{v}$ we get the maximum displacements with respect to the axial forces, which are shown in Figure 8.

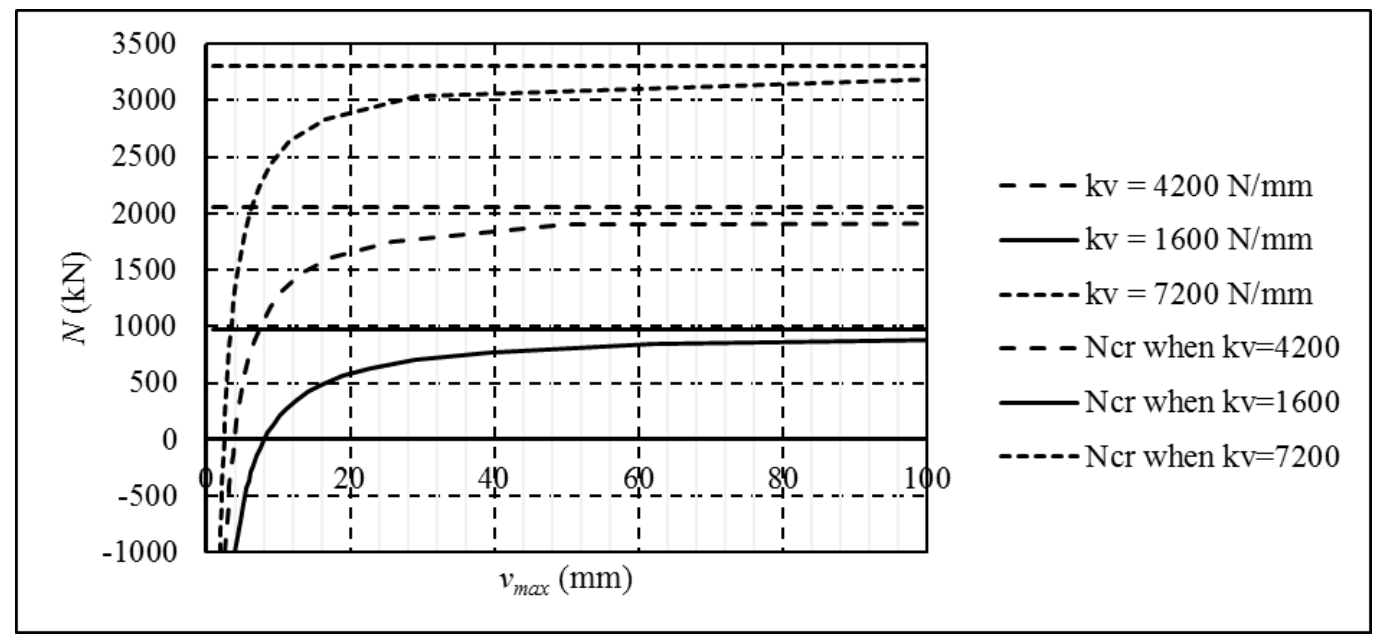

Figure 8. Maximum displacement of cantilever with respect to axial force. Positive axial load is compression.

The maximum displacement is approaching infinity when the axial load is approaching the buckling load, which is shown in Figure 8.

\section{Exact FEM formulation}

Consider a finite element of the restrained member. In Figure 9 are shown generalized forces $F_{i}(i=1, \ldots, 4)$, generalized displacements $\delta_{i}$ and notations. 


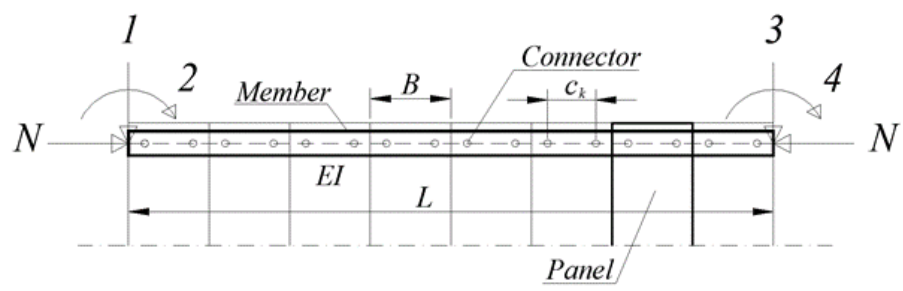

Figure 9. Restrained member.

Using the solution of the differential equation (1)

$$
v(x)=C_{1} \cos (\lambda x)+C_{2} \sin (\lambda x)+C_{3} x+C_{4}+v_{0}(x)
$$

in which is the homogenous solution with the particular solution $v_{0}(x)=0$, and using the coordinate system of Figure 9 we get

$$
[\delta]=\left[\begin{array}{l}
\delta_{1} \\
\delta_{2} \\
\delta_{3} \\
\delta_{4}
\end{array}\right]=\left[\begin{array}{c}
v(0) \\
\frac{d v(0)}{d x} \\
v(L) \\
\frac{d v(L)}{d x}
\end{array}\right]=\left[\begin{array}{cccc}
1 & 0 & 0 & 1 \\
0 & \lambda & 1 & 0 \\
\cos (\lambda L) & \sin (\lambda L) & L & 1 \\
-\lambda \sin (\lambda L) & \lambda \cos (\lambda L) & 1 & 0
\end{array}\right] \cdot\left[\begin{array}{l}
C_{1} \\
C_{2} \\
C_{3} \\
C_{4}
\end{array}\right]
$$

which is using matrix symbols

$$
[\delta]=[A] \cdot[C]
$$

This can be solved as

$$
[C]=[A]^{-1} \cdot[\delta]
$$

where 


$$
\begin{aligned}
& {[A]^{-1}=\frac{1}{D} .} \\
& {\left[\begin{array}{cccc}
-[\cos (\lambda L)-1] & \frac{1}{\lambda}\{\lambda L[\cos (\lambda L)-1]+[\sin (\lambda L)-\lambda L]\} & \cos (\lambda L)-1 & -\frac{1}{\lambda}[\sin (\lambda L)-\lambda L] \\
-\sin (\lambda L) & -\frac{1}{\lambda}[\sin (\lambda L)+\cos (\lambda L)-1] & \sin (\lambda L) & \frac{1}{\lambda}[\cos (\lambda L)-1] \\
-\lambda A_{21}^{-1} & D-\lambda A_{22}^{-1} & -\lambda A_{23}^{-1} & -\lambda A_{24}^{-1} \\
D-A_{11}^{-1} & -A_{12}^{-1} & -A_{13}^{-1} & A_{14}^{-1}
\end{array}\right](2}
\end{aligned}
$$

and

$$
D=2-2 \cos (\lambda L)-(\lambda L) \sin (\lambda L)
$$

Using the generalized forces $F_{i}(i=1, \ldots, 4)$, see Figure 9

$$
F=\left[\begin{array}{l}
F_{1} \\
F_{2} \\
F_{3} \\
F_{4}
\end{array}\right]=\left[\begin{array}{c}
-Q(0) \\
M(0) \\
Q(L) \\
-M(L)
\end{array}\right]=\left[\begin{array}{cccc}
0 & 0 & \lambda^{2} E I & 0 \\
\lambda^{2} E I & 0 & 0 & 0 \\
0 & 0 & -\lambda^{2} E I & 0 \\
-\lambda^{2} E I \cos (\lambda L) & -\lambda^{2} E I \sin (\lambda L) & 0 & 0
\end{array}\right] \cdot\left[\begin{array}{l}
C_{1} \\
C_{2} \\
C_{3} \\
C_{4}
\end{array}\right]=[B] \cdot[C]
$$

The element stiffness matrix $[K]$ is

$$
[F]=[K] \cdot[\delta] \Leftrightarrow[K]=[B] \cdot[A]^{-1}
$$

Using following notations

$$
a=4 E I / L \quad b=2 E I / L \quad c=6 E I / L^{2} \quad d=12 E I / L^{3}
$$

and Berry's functions

$$
K=\frac{(\lambda L)^{3}}{12} \frac{\sin (\lambda L)}{D} \quad H=\frac{(\lambda L)^{2}}{6} \frac{1-\cos (\lambda L)}{D}
$$




$$
\Psi=\frac{\lambda L}{4} \frac{\sin (\lambda L)-(\lambda L) \cos (\lambda L)}{D} \quad \Phi=\frac{\lambda L}{2} \frac{(\lambda L)-\sin (\lambda L)}{D}
$$

the result is

$$
[K]=\left[\begin{array}{cccc}
d K & c H & -d K & c H \\
c H & a \Psi & -c H & b \Phi \\
-d K & -c H & d K & -c H \\
c H & b \Phi & -c H & a \psi
\end{array}\right]
$$

The global stiffness matrix can be constructed using the substitution summing. If there are given only nodal forces and displacements, then the global stiffness equation can be constructed to get unknown nodal forces and displacements, and the support reactions using the normal matrix operations of FEA.

If there exist distributed loadings along the member then the particular solution $v_{0}$ must be used. The force vector $\left[F_{0}\right]$ is the force vector which is calculated using the function $v_{0}$ as the deflection of the beam and

$$
[F]=-[K] \cdot[\delta]-\left[F_{0}\right]
$$

On the other hand, the equation

$$
[\delta]+\left[\delta_{0}\right]=0
$$

is valid, where $\left[\delta_{0}\right]$ is the displacement vector calculated using the function $v_{0}$ as the displacement of the beam. So, it follows that

$$
\left\lfloor F_{e q}\right\rfloor=[K] \cdot\left[\delta_{0}\right]-\left[F_{0}\right]
$$

which are the equivalent nodal forces. This is the general approach for all particular solutions.

The function $v_{0}$ and the vectors $\left[\delta_{0}\right]$ and $\left[F_{0}\right]$ are for the uniform lateral load $q$ as:

$$
v_{0}(x)=\frac{q x^{2}}{2 E I \lambda^{2}}
$$

and 


$$
\left[\delta_{0}\right]=\left[\begin{array}{c}
0 \\
0 \\
\frac{q L^{2}}{2 E I \lambda^{2}} \\
\frac{q L}{E I \lambda^{2}}
\end{array}\right] \quad\left[F_{0}\right]=\left[\begin{array}{c}
0 \\
-\frac{q}{\lambda^{2}} \\
-q L \\
\frac{q}{\lambda^{2}}
\end{array}\right]
$$

The first equivalent nodal force is

$$
\begin{aligned}
& F_{e q 1}=d K \cdot 0+c H \cdot 0-d K \cdot \frac{q L^{2}}{2 E I \lambda^{2}}+c H \cdot \frac{q L}{E I \lambda^{2}}-0=\frac{q}{\lambda^{2} L}(-6 K+6 H) \Rightarrow \\
& F_{e q 1}=\frac{q}{\lambda^{2} L} \frac{(\lambda L)^{2}}{2}=\frac{q L}{2}
\end{aligned}
$$

and the moments are

$$
F_{e q 2}=\frac{q}{\lambda^{2}}\{-3 H+2 \Phi+1\} \quad F_{e q 4}=\frac{q}{\lambda^{2}}\{-3 H+4 \psi-1\}
$$

and the result is

$$
\left[F_{e q}\right]=\left[\begin{array}{c}
\frac{q L}{2} \\
\frac{q}{\lambda^{2}}\left\{\frac{\frac{1}{2}(\lambda L)^{2}+\frac{1}{2}(\lambda L)^{2} \cos (\lambda L)-(\lambda L) \sin (\lambda L)}{D}+1\right\} \\
\frac{q L}{2} \\
-\frac{q}{\lambda^{2}}\left\{\frac{\frac{1}{2}(\lambda L)^{2}+\frac{1}{2}(\lambda L)^{2} \cos (\lambda L)-(\lambda L) \sin (\lambda L)}{D}+1\right.
\end{array}\right\}
$$

The similar approach can be used for other load cases to get the equivalent nodal forces.

Consider next the FEM formulation using the solution of the differential equation (3).

$$
v(x)=\bar{C}_{1} \cosh (\bar{\lambda} x)+\bar{C}_{2} \sinh (\bar{\lambda} x)+\bar{C}_{3} x+\bar{C}_{4}+v_{0}(x)
$$

The generalized displacements $\delta_{i}(i=1, \ldots, 4)$ are 


$$
[\delta]=\left[\begin{array}{l}
\delta_{1} \\
\delta_{2} \\
\delta_{3} \\
\delta_{4}
\end{array}\right]=\left[\begin{array}{c}
v(0) \\
\frac{d v(0)}{d x} \\
v(L) \\
\frac{d v(L)}{d x}
\end{array}\right]=\left[\begin{array}{cccc}
1 & 0 & 0 & 1 \\
0 & \bar{\lambda} & 1 & 0 \\
\cosh (\bar{\lambda} L) & \sinh (\bar{\lambda} L) & L & 1 \\
\bar{\lambda} \sinh (\bar{\lambda} L) & \bar{\lambda} \cosh (\bar{\lambda} L) & 1 & 0
\end{array}\right] \cdot\left[\begin{array}{c}
\bar{C}_{1} \\
\bar{C}_{2} \\
\bar{C}_{3} \\
\bar{C}_{4}
\end{array}\right]
$$

which is written using matrix symbols

$$
[\delta]=[\bar{A}] \cdot[\bar{C}]
$$

This can be solved as

$$
[\bar{C}]=[\bar{A}]^{-1} \cdot[\delta]
$$

where

$$
\begin{aligned}
& {[\bar{A}]^{-1}=\frac{1}{\bar{D}} .} \\
& {\left[\begin{array}{cccc}
\cosh (\bar{\lambda} L)-1 & \frac{1}{\bar{\lambda}}[\bar{\lambda} L \cosh (\bar{\lambda} L)-\sinh (\bar{\lambda} L) & -[\cosh (\bar{\lambda} L)-1] & \frac{1}{\bar{\lambda}}[\sinh (\bar{\lambda} L)-\bar{\lambda} L] \\
-\sinh (\bar{\lambda} L) & -\frac{1}{\bar{\lambda}}[\bar{\lambda} L \sinh (\bar{\lambda} L)-\cosh (\bar{\lambda} L)+1] & \sinh (\bar{\lambda} L) & -\frac{1}{\bar{\lambda}}[\cosh (\bar{\lambda} L)-1] \\
-\bar{\lambda} \cdot \bar{A}_{21}^{-1} & \bar{D}-\bar{\lambda} \cdot \bar{A}_{22}^{-1} & -\bar{\lambda} \cdot \bar{A}_{23}^{-1} & -\bar{\lambda} \cdot \bar{A}_{24}^{-1} \\
\bar{D}-\bar{A}_{11}^{-1} & -\bar{A}_{12}^{-1} & -\bar{A}_{13}^{-1} & -\bar{A}_{14}^{-1}
\end{array}\right]}
\end{aligned}
$$

and

$$
\bar{D}=2 \cosh (\bar{\lambda} L)-\bar{\lambda} L \sinh (\bar{\lambda} L)-2
$$

Using the generalized forces $F_{i}(i=1, \ldots, 4)$ 


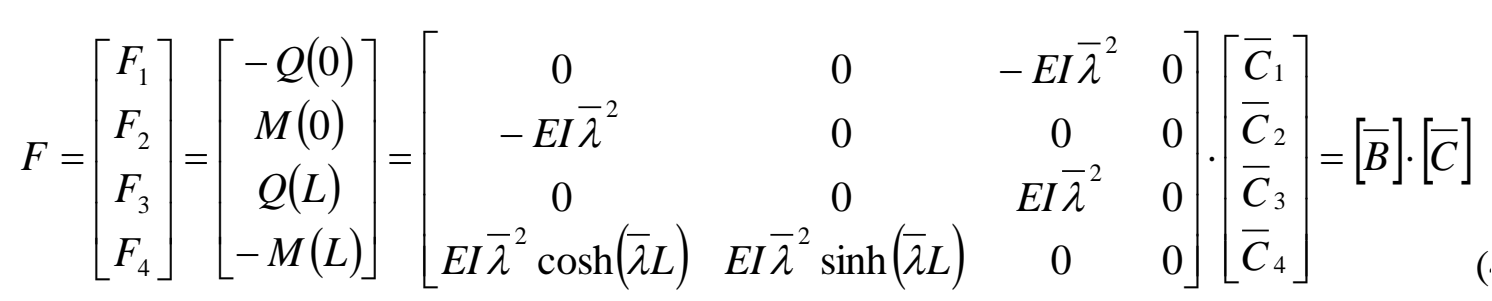

The element stiffness matrix is

$$
[F]=[\bar{K}] \cdot[\delta] \Leftrightarrow[\bar{K}]=[\bar{B}] \cdot[\bar{A}]^{-1}
$$

The result is

$$
\begin{aligned}
& {[\bar{K}]=\frac{E I \bar{\lambda}}{\bar{D}} .}
\end{aligned}
$$

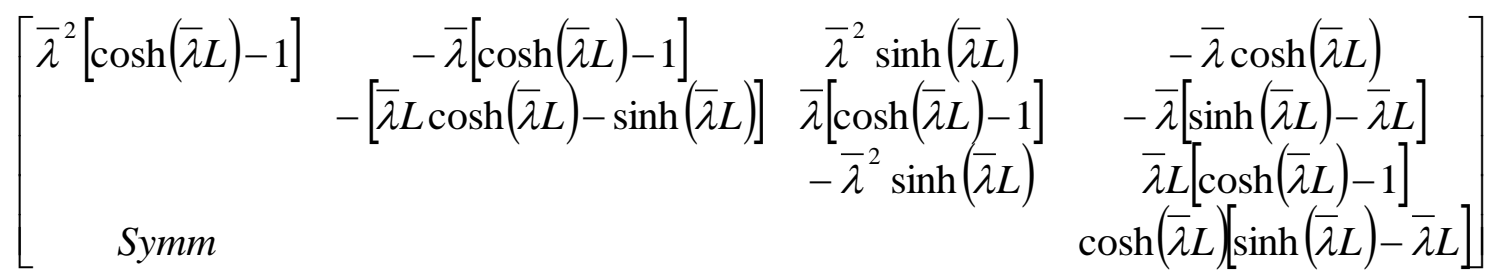

Formally the stiffness matrixes are the same as those for axially loaded member without restraint. The differences appear in the definitions of parameters $\lambda$ and in the definition of the shear force $Q$.

As an example consider a cantilever column (CFRHS $300 * 200 * 8, E=210000 \mathrm{MPa}$, $I=5042 \mathrm{~cm}^{4}, L=9.3 \mathrm{~m}$ ) with the panels missing both sides at the bottom part of the column in the range $x=0-3.3 \mathrm{~m}$ simulating a door opening of the wall. The column is loaded by the axial force $N$ at the top. At the top part of the column are panels ( $B=1.2$ $\mathrm{m}, c_{1}=1 \mathrm{~m}$, one pair of connectors per end of the panel) at both sides of the column, see Figure 10. The shear stiffness of one connector is $2.1 \mathrm{kN} / \mathrm{mm}$ and the total spring stiffness in the analysis is $k_{v}=4.2 \mathrm{kN} / \mathrm{mm}$. There exist two connectors at the same level of the column. The flexural buckling loads in the plane of the panels (weak axis buckling) and the corresponding buckling lengths are calculated firstly using the exact FEM and then using the approximative FEM formulation with polynomials as the shape functions, Timoshenko's model. 


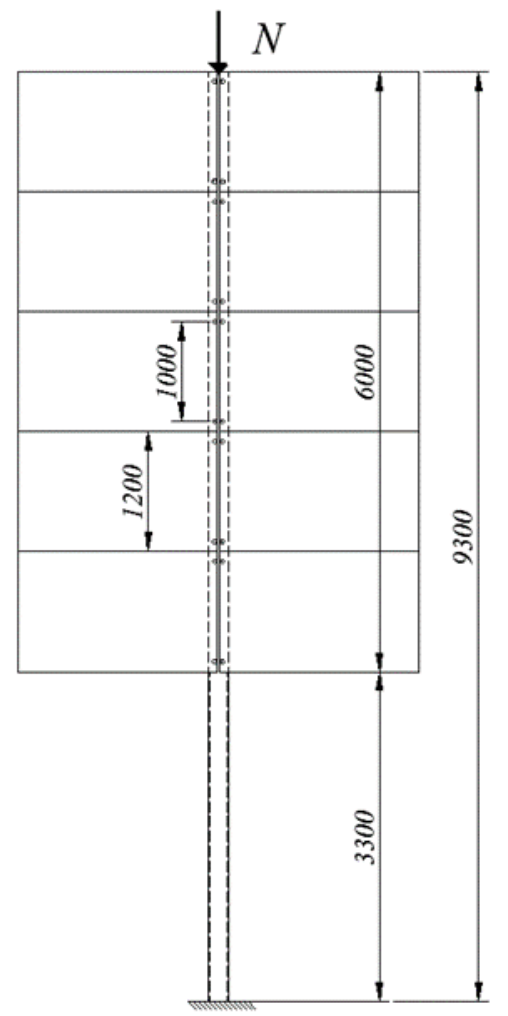

Figure 10. Cantilever column with door opening in sandwich panels, dimensions in mms.

Using the exact FEM both for the lower and for the upper part of the column only two elements are needed to solve the problem. The exact FEM for the column without panels is the same as above but using the stiffness of connectors the value $k_{v}=0$. The global stiffness matrix is constructed using the normal substitution summing. After substitution summing and use of the boundary conditions, we end up to the final $4 \times 4$ stiffness matrix $\left[K_{s y s}\right]$. The final degrees of freedoms are two displacements and two rotations. Next, solve numerically the lowest positive $N_{c r}$ from the equation $\operatorname{det}\left[K_{s y s}\right]=$ 0 . The solution is $N_{c r}=1869.1 \mathrm{kN}$ and the corresponding buckling length is $L_{c r}=2.365$ $\mathrm{m}$. Consider next the same case using the polynomial shape functions in the FEM formulation. The panel is modeled as rigid, the connectors as horizontal springs and the free ends of panels are supported in the vertical direction. The results are shown in Table 3.

Table 3. Convergence of approximative FEM solution.

\begin{tabular}{ccc}
\hline $\begin{array}{c}\text { Number of elements using polynomial } \\
\text { shape functions per 3.3 and } 6.0 \mathrm{~m}\end{array}$ & $N_{c r}(\mathrm{kN})$ & $\begin{array}{c}\text { Ratio of approximative and exact } \\
\text { solution }\end{array}$ \\
\hline 1 & 1870.4 & 1.0007 \\
2 & 1868.7 & 0.99979 \\
3 & 1868.6 & 0.99973 \\
4 & 1868.6 & 0.99973 \\
Exact solution & 1869.1 & 1.00 \\
\hline
\end{tabular}


It can be seen in Table 3, that in this case the convergence with polynomial shape functions is very fast. The exact solution $N_{c r}$ in Table 3 is a little bit larger than the converged solution with polynomials, because in that solution the shear deformations of the members are considered meaning not so stiff elements than in the exact solution. The differences in the values are not large.

\section{Conclusions}

It is shown by examples that the presented analytic method is suitable for the approximation of the flexural displacements of the member, which is restrained by the sandwich panels. The basic assumptions of the method are outlined. Examples of buckling cases and $P-\delta$ analyses are given in order to demonstrate the method in practical applications. The exact FEM formulation is given for the analyses of the cases where the properties of the member change along its axis.

It is believed by the author that the restraint effects of the sandwich panels include the unused potential in design of economic and environmental effective steel structures. This seems to be true in the cases, where the buckling strength of the member in the plane of the panels is critical, as is in the presented example of the simply supported member.

In the future some cases may be analyzed in the fire cases in order to see if the restraint effect may stay in fire. This is possible, if the stiffness values and the resistances of all entities are available at elevated temperatures.

\section{Acknowledgements}

The financial support of European Commission and Research Programme of the Research Fund for Coal and Steel, project: Steel cladding systems for stabilization of steel buildings in fire, 751583 - STABFI - RFCS-2016, is gratefully acknowledged.

\section{References}

[1] R. Baehre, Th. Ladwein. Diaphragm action of sandwich panels. Journal of Constructional Steel Research 31, 305-316, 1994. https://doi.org/10.1016/0143974X(94)90015-9

[2] E. Bryan. The stressed skin design of steel buildings. Constrado monographs. London: Crosby Lockwood Staples, 1973.

[3] R. Clough, J. Penzien. Dynamics of Structures. New York, McGraw-Hill Inc, 1974.

[4] J. Davies, E. Bryan. Manual of Stressed Skin Diaphragms Design. Granada, London, 1982.

[5] EASIE, FP7/NMP2-SE-2008, Grant agreement No 213302, 2008.

[6] ECCS Publication No. 88: European recommendations for the application of metal sheeting acting as a diaphragm, 1995.

[7] ECCS, European Recommendations on the Stabilization of Steel Structures by Sandwich Panels, ECCS TC7 TWG 7.9: Sandwich panels and Related structures, 
CIB Working Commission: W056 Sandwich Panels, ECCS/CIB Joint Committee, 2014.

[8] M. Eisenberger, D. Yankelevsky, J. Clastornik. Stability of beams on elastic foundation, Computers \& Structures 24, 135-139, 1986. https://doi.org/10.1016/0045-7949(86)90342-1

[9] EN 1993-1-3, Eurocode 3. Design of steel structures. Part 1-3: General rules. Supplementary rules for cold-formed members and sheeting. 2006.

[10] P. Friberg. Beam element matrices derived from Vlasov's theory of open thinwalled elastic beams. International Journal for Numerical Methods in Engineering 21, 1205-1228, 1985. https://doi.org/10.1002/nme.1620210704

[11] T. Hannula. Parametric review with stressed skin for stabilization. Ms thesis, Faculty of business and built environment, Tampere University of Technology, Tampere, Finland, 2018. http://urn.fi/URN:NBN:fi:tty-201811212679

[12] E. Hedman-Pétursson. Column Buckling with Restraint from Sandwich Wall Elements. Department of Civil and Mining Engineering, Division of Steel Structures, Lulea University of Technology, 2001. urn:nbn:se:ltu:diva-18056

[13] M. Heinisuo. Kimmoisalla alustalla olevan palkin ratkaisusta. Tampereen teknillinen korkeakoulu, Rakennusstatiikka, Raportti No 7, Tampere, 1984. (in Finnish)

[14] M. Heinisuo. An exact finite element technique for layered beams, Computers \& Structures, Vol 30. No. 3. 615-622, 1988. https://doi.org/10.1016/00457949(88)90297-0

[15] M. Hetenyi. Beams on elastic foundation. Ann Arbor: The University of Michigan Press, 1979.

[16] T. Höglund. Stabilisation by stressed skin diaphragm action. The Swedish Institute of Steel Construction, Publication 174, 2002.

[17] Z. Ma, J. Havula, M. Heinisuo. Numerical analysis of steel columns stabilized by sandwich panels. Rakenteiden Mekaniikka (Journal of Structural Mechanics), Vol. 50, No 2, 97-113, 2017. https://doi.org/10.23998/rm.65309

[18] J. Przemieniecki. Theory of Matrix Structural Analysis. New York, McGraw-Hill Book Company, 1968.

[19] S. Timoshenko, J. Gere. Theory of Elastic Stability, McGraw-Hill Book, New York, 1961.

[20] F. Williams, W. Wittrick. An automatic computational procedure for calculating natural frequencies of skeletal structures, Int. J. Mech. Sci. 12, 781-791, 1970. https://doi.org/10.1016/0020-7403(70)90053-6

Markku Heinisuo

Tampere University

Korkeakoulunkatu 10, 33720 Tampere, Finland

markku.heinisuo@tuni.fi 